\title{
A single genetic locus associated with pediatric fractures: A genome-wide association study on 3,230 patients
}

\author{
ROOPE PARVIAINEN $^{1}$, SINI SKARP ${ }^{2,3}$, LINDA KORHONEN ${ }^{1}$, WILLY SERLO ${ }^{1}$, \\ MINNA MÄNNIKKÖ $^{2}$ and JUHA-JAAKKO SINIKUMPU ${ }^{1}$
}

\begin{abstract}
${ }^{1}$ Department of Children and Adolescents, Oulu Childhood Fracture and Sports Injury Study, Research Unit for Pediatrics, Pediatric Neurology, Pediatric Surgery, Child Psychiatry, Dermatology, Clinical Genetics, Obstetrics and Gynecology, Otorhinolaryngology and Ophthalmology (PEDEGO), Oulu Medical Research Center (MRC), University of Oulu and Oulu University Hospital, FI-90029 Oulu; ${ }^{2}$ Northern Finland Birth Cohort, Faculty of Medicine, University of Oulu;

${ }^{3}$ Center for Life Course Health Research, Faculty of Medicine, University of Oulu, FI-90014 Oulu, Finland
\end{abstract}

Received July 13, 2019; Accepted April 29, 2020

DOI: $10.3892 / \mathrm{etm} .2020 .8885$

\begin{abstract}
The understanding of the biological and environmental risk factors of fractures in pediatrics is limited. Previous studies have reported that fractures involve heritable traits, but the genetic factors contributing to the risk of fractures remain elusive. Furthermore, genetic influences specific to immature bone have not been thoroughly studied. Therefore, the aim of the present study was to identify genetic variations that are associated with fractures in early childhood. The present study used a prospective Northern Finland Birth Cohort (year 1986; $n=9,432$ ). The study population was comprised of 3,230 cohort members with available genotype data. A total of 48 members of the cohort (1.5\%) had in-hospital treated bone fractures during their first 6 years of life. Furthermore, individuals without fracture $(n=3,182)$ were used as controls. A genome-wide association study (GWAS) was performed using a frequentist association test. In the GWAS analysis, a linear regression model was fitted to test for additive effects of single-nucleotide polymorphisms (SNPs; genotype dosage) adjusting for sex and performing population stratification using genotypic principal components. Using the GWAS analysis, the present study identified one locus with a significant association with fractures during childhood on chromosome 10 (rs112635931) and six loci with a suggested implication. The lead SNP rs112635931 was located near proline- and serine-rich 2 (PROSER2) antisense
\end{abstract}

Correspondence to: Mr. Roope Parviainen, Department of Children and Adolescents, Oulu Childhood Fracture and Sports Injury Study, ResearchUnitforPediatrics,Pediatric Neurology,Pediatric Surgery,Child Psychiatry, Dermatology, Clinical Genetics, Obstetrics and Gynecology, Otorhinolaryngology and Ophthalmology (PEDEGO), Oulu Medical Research Center (MRC), University of Oulu and Oulu University Hospital, P.O. Box 5, FI-90029 Oulu, Finland

E-mail: roope.parviainen@student.oulu.fi

Key words: fractures, risk, children, genetics, genome-wide association study, cohort
RNA 1 (PROSER2-AS1) and PROSER2, thus suggesting that these may be novel candidate genes associated with the risk of pediatric fractures.

\section{Introduction}

The incidence of fractures is highest among the young and older populations (1). Of note, fractures in pediatric patients are common injuries that result in pain, as well as short- and long-term morbidity; it has been estimated that $27-50 \%$ of all individuals experience a fracture prior to turning 18 years old $(2,3)$. Goulding et al $(4)$ estimated the risk of recurrent fracture to be $12-20 \%$ in pediatrics patients aged $<16$ years. Furthermore, these figures may indicate that pediatric patients who do not have any diseases affecting bone integrity may still be at risk to suffer a bone fracture or even recurrent fractures (2-4). The causes for this may arise from the diet, preferred free-time activities or sex, but may also be linked to genetic factors (3).

The causes and etiopathogenetic pathways of pediatric fractures are not well studied (5). It has been previously reported that bone-associated and bone-independent factors contribute to the fracture risk $(2,6)$. Furthermore, bone mineral content (BMC) and bone mineral density (BMD) have been indicated to be highly heritable in genetic studies (7-11). Mora and Gilsanz (12) revealed that $80 \%$ of the variance of the $\mathrm{BMC}$ is influenced by genetics. Furthermore, BMD has been reported to be a risk factor for fractures in adults (1), but there is limited research on pediatric fractures. Previous studies have linked a low BMD to increased fracture risk during childhood $(3,13)$; however, in other studies, this association was not identified $(6,14)$. In adults, genome-wide association studies (GWAS) have identified genes from $\geq 11$ different chromosomes (chrs) that may affect BMD either locally or throughout the body (15-21). However, with regards to pediatrics, only few studies have reported on genetic loci that influence BMD or cortical bone thickness $(7,8)$. Furthermore, the heritability of the fractures and BMD differ, and thus, it has been suggested that fracture risk should be analyzed independently of BMD (22). 
BMD and BMC are useful when examining the factors underlying fractures $(23,24)$; however, their association with fractures in childhood remains to be fully elucidated. Therefore, the present study focused on fractures rather than BMD or BMC. To the best of our knowledge, only few studies have examined the association between genetics and childhood fracture risk. Therefore, the aim of the present study was to identify whether any genetic loci are associated with fractures prior to the age of 7 years using GWAS.

\section{Patients and methods}

Patient population. The study population was a part of the Northern Finland Birth Cohort 1986 (NFBC1986) that originally included all pregnant females $(n=9,362)$ living in the geographic area of the two northernmost provinces of Finland with expected date of delivery between 1st July 1985 and 30th June 1986. These mothers and their live-born infants $(n=9,432)$ were followed up regularly since pregnancy. Immigration during this period to northern Finland was minimal, and therefore, the cohort was predominantly Finnish (25). The detailed description of the cohort is provided in the study published by Järvelin et al (26) and on the NFBC website (27).

Of the cohort population, 6,721 consented to the use of their information for research purposes. The information for the individuals who did not provide consent was not available. Study subjects with diseases of the bone, including osteogenesis imperfecta, or possible bone-affecting malignancies, were excluded from the original study population $(n=3)$. Informed consent from the parents was provided orally during antenatal clinic visits. At the 16-year follow-up (in 2001), the cohort participants and their parents provided written informed consent for the use of all of their collected data for scientific purposes. The participation was voluntary.

Genotype data. Genotypes were determined for the cohort members who had provided consent and their blood samples were used for this purpose. The genotype data were available for 3,230 individuals, including 48 cases who had a fracture and 3,182 controls without fractures. Genotyping was performed using the Illumina HumanOmniExpressExome v1.2 bead chip (Illumina, Inc.) and imputation was performed using the $1000 \mathrm{G}$ phase 3 reference panel (28). After quality control (QC), the study included 3,515,000 single-nucleotide polymorphisms (SNPs) (28). The QC process is described in detail in a previous study (28). The data was controlled based on genotype call rate, minor allele frequency, multidimensional scaling outliers, relatedness, heterozygosity rate and Hardy-Weinberg Equilibrium. The imputation quality threshold was 0.4 . The flow chart for inclusion is presented in Fig. 1.

Fractures. The National Hospital Discharge Register (NHDR) provided the data on the fractures (the name of the register has changed and it is now known as Care Register for Health Care). The register contains information on all in-hospital treated fractures with diagnostic codes and discharge dates. The NHDR is coordinated by the Finnish institute for health and welfare and it contains all hospital admissions in Finland (29). This register is the oldest nationwide discharge
Table I. Reported ICD-9 codes.

\begin{tabular}{ll}
\hline ICD-9 code & \multicolumn{1}{c}{ Explanation } \\
\hline 800 & Fracture of vault of skull \\
802 & Fracture of facial bones \\
810 & Fracture of clavicle \\
812 & Fracture of humerus \\
813 & Fracture of radius or ulna \\
816 & Fracture of one or more phalanges of the hand \\
821 & Fracture of other or unspecified parts of femur \\
823 & Fracture of tibia or fibula
\end{tabular}

ICD, International Classification of Diseases.

register worldwide and thoroughly covers acute injuries of the population (annual coverage of injuries, $\sim 100 \%$ ) and classifies these injuries accurately (annual accuracy, >95\%) (30).

In the study area, pediatric fractures are exclusively treated in public hospitals and it is mandatory to send information of these injuries to the NHDR. During the study period, out-patient visits were not recorded in the NHDR and any patients treated outside the hospital were not included. Simple fractures, including non-displaced fracture of wrist or foot, were not included in the NHDR, as these are treated by general practitioners in primary healthcare centers. All the in hospital treated fractures were recognized for the analysis and the patients were classified according to whether they had a fracture or not. The International Classification of Diseases, version-9 (ICD-9) was used to identify the fractures (31). If the patient had several hospitalizations with the same ICD code, the fractures were determined to be caused by $\geq 2$ separate injuries if there was an interval of $\geq 6$ months between the hospitalization dates. The ICD-9 codes reported for the fractures are presented in Table I.

The cause of the fracture may also be included in the NHDR data. Out of the 48 individuals who had a fracture in the population of the present study, 37 had a reported mechanism. Furthermore, 15 patients had low-energy accidents, including falling onto a plane, tripping, slipping or falling from a height of $<1 \mathrm{~m}$. For the other fractures, it was not possible to reliably determine the energy involved in the injury.

Data analyses. The association between specific genomic regions and fractures was assessed in the GWAS using the frequentist association test of the SNPTEST analysis software (version 2.5.2; University of Oxford). In the GWAS analysis, a linear regression model was fitted to test for additive effects of SNPs (genotype dosage) adjusting for sex and population stratification using four principal components. Sex and the first four principal components were used as covariates.

The post-GWAS QC included the following steps: Minor allele frequency $<0.05$, deviation from Hardy-Weinberg equilibrium $\mathrm{P}$-value $<1 \times 10^{-7}$. The generally accepted limit of $\mathrm{P}<5 \times 10^{-8}$ for genome-wide significance and $\mathrm{P}<5 \times 10^{-5}$ for suggestive evidence of association were used. Under the null hypothesis, the threshold where one false positive is expected per genome scan is called the suggestive level (32). 


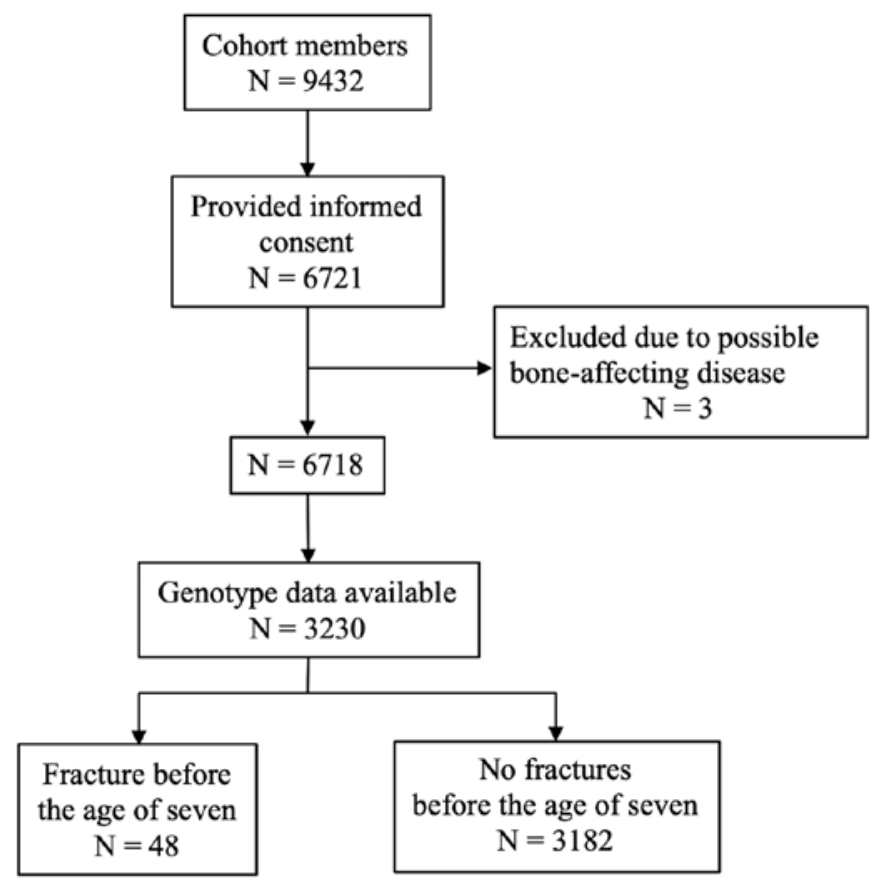

Figure 1. Flow chart of the inclusion criterion.

Manhattan and Quantile-Quantile (Q-Q) plots of the results were generated using $\mathrm{R}$ (version 3.5.0) and the qqman package (version 0.1.7) (33). The closest coding and non-coding genes in the identified loci were determined using the University of California Santa Cruz Genome Browser (December 2013), which is a graphical viewing tool of the human genome assembly. The genes were visualized using the LocusZoom visualization software (version 1.4; University of Michigan).

After GWAS, the lead SNPs were analyzed using the public databases GWAS database (GWASdb) (34), GWAS catalog (35), The Genotype-Tissue Expression (GTEx) Portal (36) and RegulomeDB (37). GWASdb and GWAS catalog are databases of genetic variants identified in published GWASs. GTEx Portal is database of tissue-specific gene expression. RegulomeDB is used to annotate variants to known and predicted regulatory elements. Database searches were performed for the locus surrounding the lead SNP (chr10:11491165-12291165). Associated loci were compared with summary statistics from two studies available on the Genetic Factors for Osteoporosis Consortium (GEFOS) website $(38,39)$. Trajanoska et al $(38)$ included 25 cohorts and had discovery dataset of 37,857 fracture cases and 227,116 controls composed. All the subjects were adults and predominantly of European descent (38). Medina-Gomez et al (39) was meta-analysis of total body bone mineral density and included 66,628 individuals, both children and adults of mostly European descent (39).

\section{Results}

Fracture demographics. In total, 48 patients had a fracture prior to the age of 7 and one of these patients had two fractures. The characteristics of the study population are presented in Table II. Of the 48 subjects with fractures, 16 (33.3\%) were female and $32(66.7 \%)$ were male. The average age at the time
Table II. Characteristics of the study population.

\begin{tabular}{lc}
\hline Characteristic & Value \\
\hline Sex (female/male) & $16 / 32$ \\
Fracture & $1,648 / 1,534$ \\
No fracture & $4.05(0-6)$ \\
Average age at the time of the & \\
fracture (years) & \\
Average BMI at 7 years of age ${ }^{\mathrm{a}}$ & \\
Fracture & $16.6(13.1-20.8)$ \\
No fracture & $(10.4-34.8)$ \\
Location of fracture & \\
Skull & 1 \\
Facial bones & 1 \\
Vertebral column & 1 \\
Clavicle & 2 \\
Humerus & 10 \\
Radius or ulna & 14 \\
One or more phalanges of the hand & 4 \\
Other or unspecified parts of femur & 4 \\
Tibia or fibula & 12 \\
\hline
\end{tabular}

an the cohort, the body height and weight were recorded when the subjects turned 7 years old. Values are expressed as the average (range) or n. BMI, body mass index.

of fracture was 4.05 years (range $0-6$ years). Furthermore, the fractures included one cervical spine fracture (ICD-9 806), two skull fractures (ICD-9 800), eight lower limb fractures (ICD-9 821, 823) and 37 upper limb fractures (ICD-9 810, 812, 813 and 816$)$.

Genetics of fractures. The GWAS analysis identified one locus with a significant association and six loci with a suggestive result (Table III; Fig. 2). The Q-Q plot of the GWAS is presented in Fig. 3.

The lead SNP rs112635931 with the most significant GWA was located within the proline- and serine-rich 2 (PROSER2) and PROSER2-antisense RNA 1 (AS1) genes (Fig. 4). The lead SNP (rs9827298) with results suggestive of an association with fracture risk is located in the intergenic region near the leucine-rich repeat neuronal 1 (LRRN1), SET domain and mariner transposase fusion gene (SETMAR) and sulfatase-modifying factor 1 (SUMF1) genes (Fig. 5). According to GWASdb and GWAS catalog analyses, no genome-wide significant associations with any other trait were reported for either of the SNPs. They were also not present in the RegulomeDB. In addition, the present study did not identify any SNPs associated with other phenotypes that may be in linkage disequilibrium with the lead SNP.

The SNP rs374077976 was present in GWASdb and this SNP has been associated with total cholesterol in a European population (40). Furthermore, the SNP rs111299584 in chr 3 is considered to likely affect the binding of transcription factors to DNA according to the RegulomeDB. Furthermore, SNPs rs374077976, rs17762577, rs41316954 and rs35417231 were 


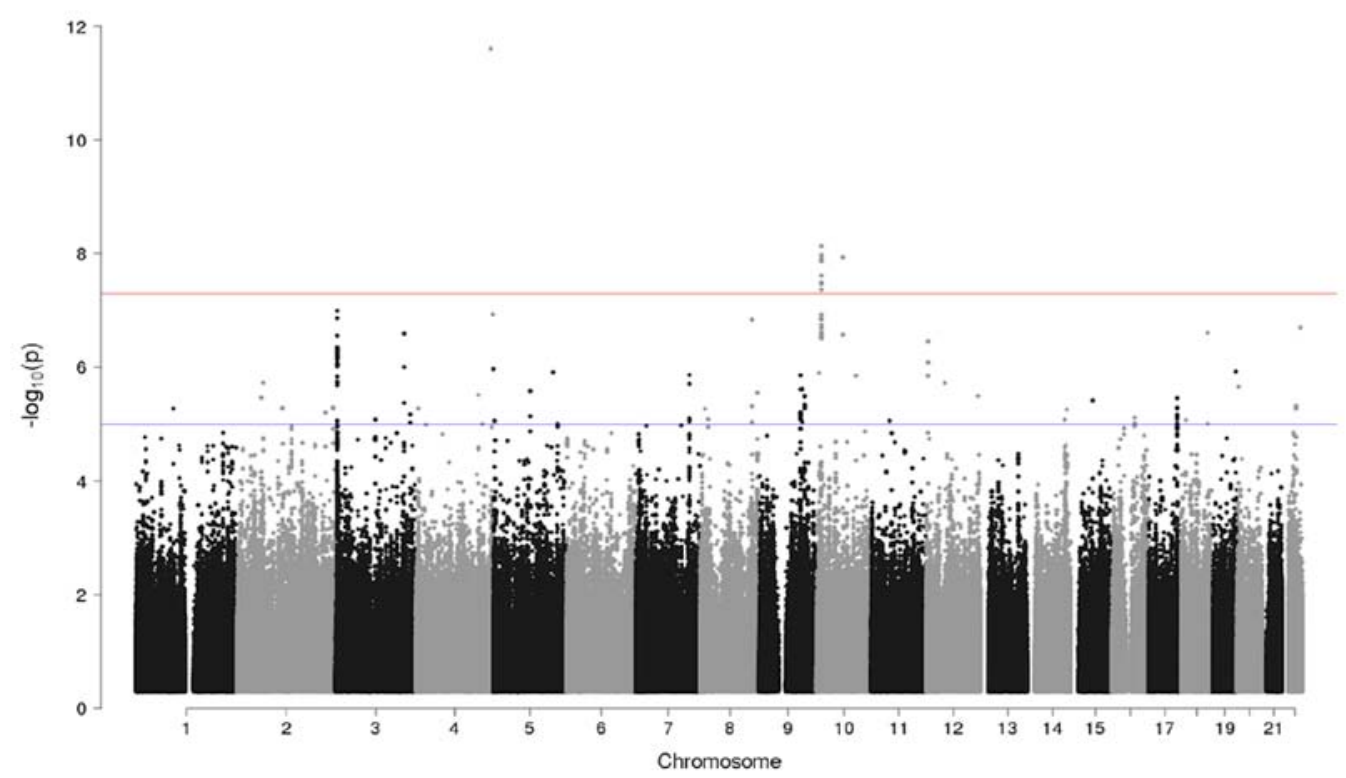

Figure 2. Manhattan plot of the genome-wide association study. The red line refers to the level of statistical significance $\left(\mathrm{P}=5 \times 10^{-8}\right)$ and blue line marks the level of suggestive significance.

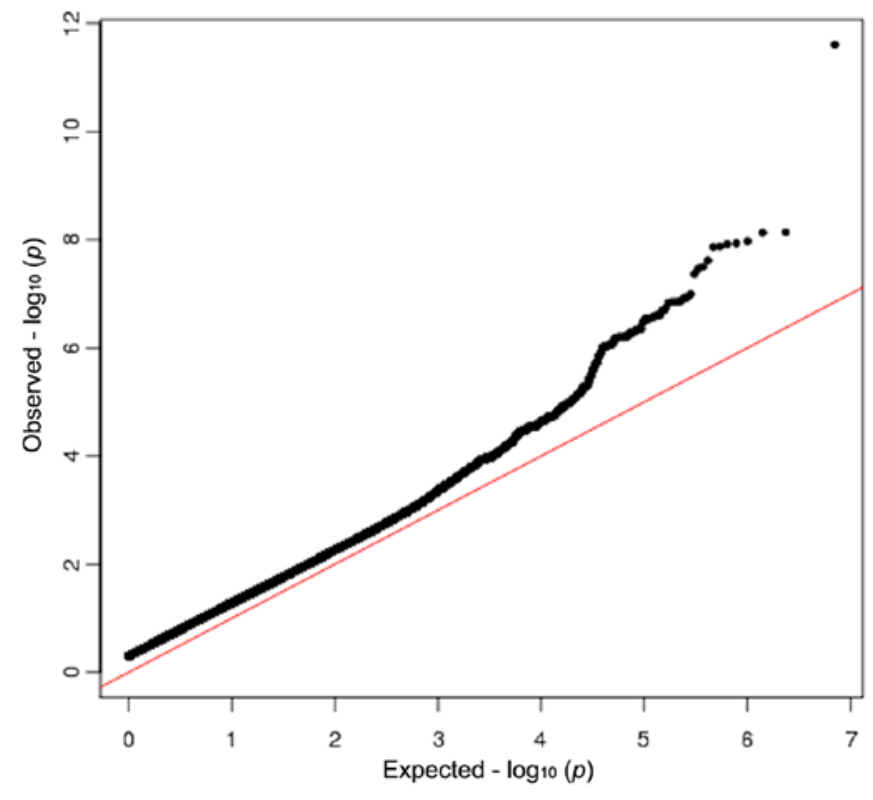

Figure 3. Quantile-quantile plot from the genome-wide association study analysis. The red line represents the case where the compared distributions are similar.

categorized to have minimal evidence of DNA binding of transcription factors in the RegulomeDB. In addition, none of the studied loci were replicated in the summary statistics of the two GEFOS studies $(35,38)$.

\section{Discussion}

The results of the present study are important, as the understanding of the genetic associations with childhood fractures is limited, despite the fact that these are common injuries. Recent studies have identified certain risk factors for pediatric fractures both at the micro-level (for example, maternal smoking associates with childhood fractures) (41) and the macro-level (for example, weather conditions associates with upper extremity fractures) (42). However, further studies examining childhood fractures are required (43). Furthermore, previous studies focusing on the possible genetic origins of fractures in pediatric patients without diseases affecting the bone are limited; to the best of our knowledge, the present study provides novel GWAS data on fractures in early childhood.

Previous studies have reported that BMC and BMD are highly heritable (7-11). Furthermore, GWAS have revealed $\geq 11$ different chromosomes that affect BMD in adults (15-21). Chesi et al (8) identified five loci that influence pediatric BMD at multiple skeletal sites. Duren et al (7) identified three genomic regions (2p25.2, 3p25.3 and 17q21.2) with linkage to cortical bone thickness in 10-year-old children (7). These regions contain candidate genes, certain of which have been associated with adult bone mass in other studies (17,44-46). Chesi et al (47) performed a GWAS of areal BMD and BMC and indicated that two loci had a statistically significant association with the BMD and BMC of the distal radius. Furthermore, the bone mass phenotype has been revealed to influence the development of osteoporosis and it is suggested that genetics may impact osteoporotic fracture risk (22).

The primary result of the present study was the statistically significant association between the lead SNP rs112635931 and bone fractures in preschool pediatric patients. Furthermore, SNP rs112635931 was located within the PROSER2 gene, and according to expression quantitative trait loci (eQTL) of the GTEx database, it may regulate the expression of the PROSER2-AS1 gene. Furthermore, the PROSER2 protein is abundant throughout different organs in the human body, including the bone marrow and muscle tissues, based on the GTEx and Human Protein atlas databases $(48,49)$. The expression of PROSER2 protein was also reported to be present in two mouse osteoblast cell lines according to data in the Gene Expression Omnibus database $(50,51)$; however, the exact function of PROSER2 remains to be fully elucidated. 


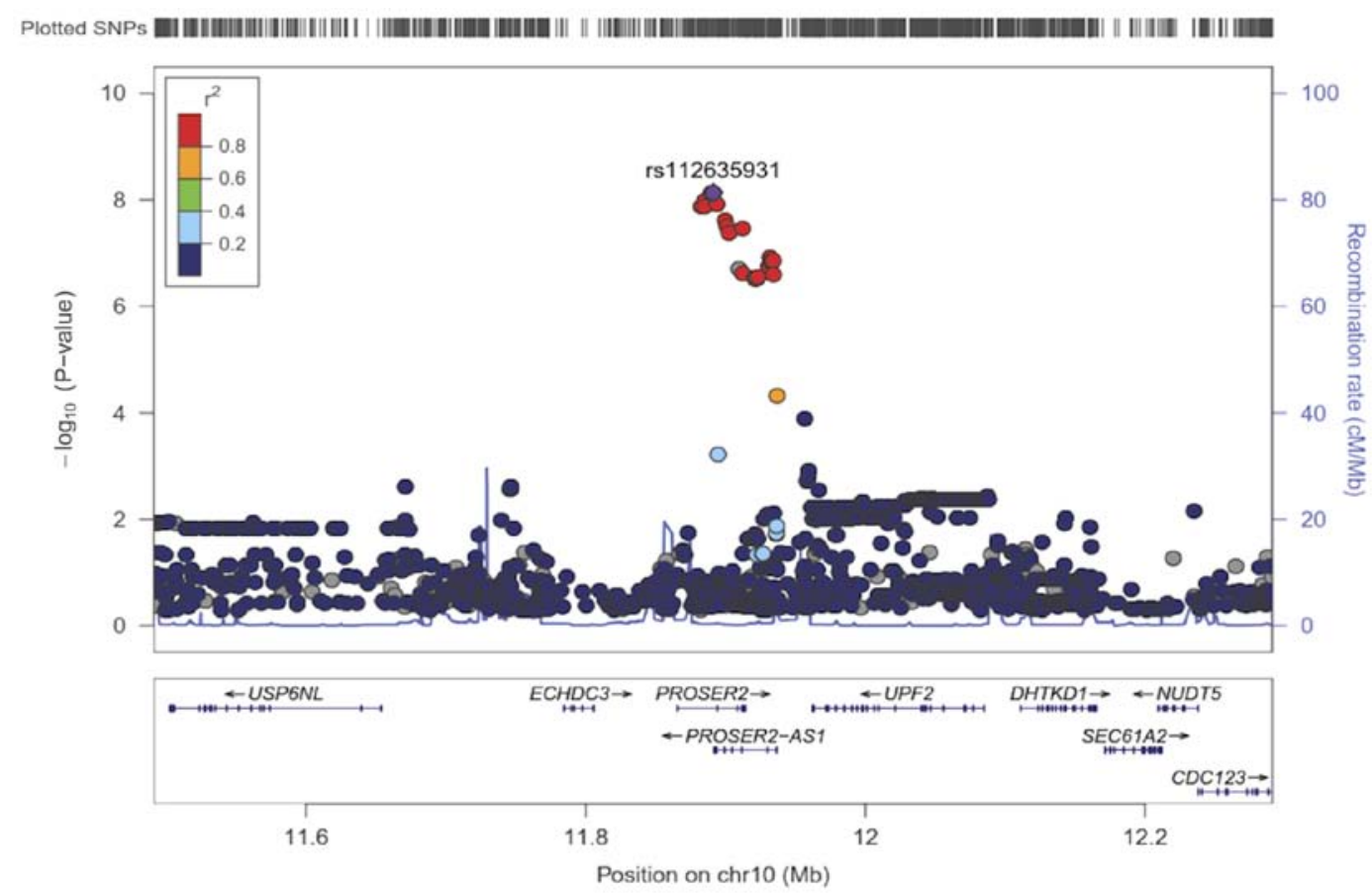

Figure 4. LocusZoom of the rs112635931, Chr 10:11891165, P=7.28x10-9. Each dot represents the P-value of the SNP obtained from the genome-wide association study. The color of the dot represents the linkage disequilibrium $\left(\mathrm{r}^{2}\right)$, which means that the frequency of association of the different alleles is higher than what would be expected if the loci were independent and associated randomly. Chr, chromosome; SNP, single-nucleotide polymorphism; PROSER2-AS1, proline- and serine-rich 2-antisense RNA 1; USP6NL, Ubiquitin Specific Peptidase 6 N-terminal Like; ECHDC3, Enoyl-CoA Hydratase Domain Containing 3 ; UPF2, up-frameshift 2; DHTKD1, Dehydrogenase E1 And Transketolase Domain Containing 1; SEC61A2, Selenocystein 61 Translocon Subunit Alpha 2; NUDT5, Nudix Hydrolase 5, Nudix Hydrolase 5; CDC123, Cell Division Cycle 123.

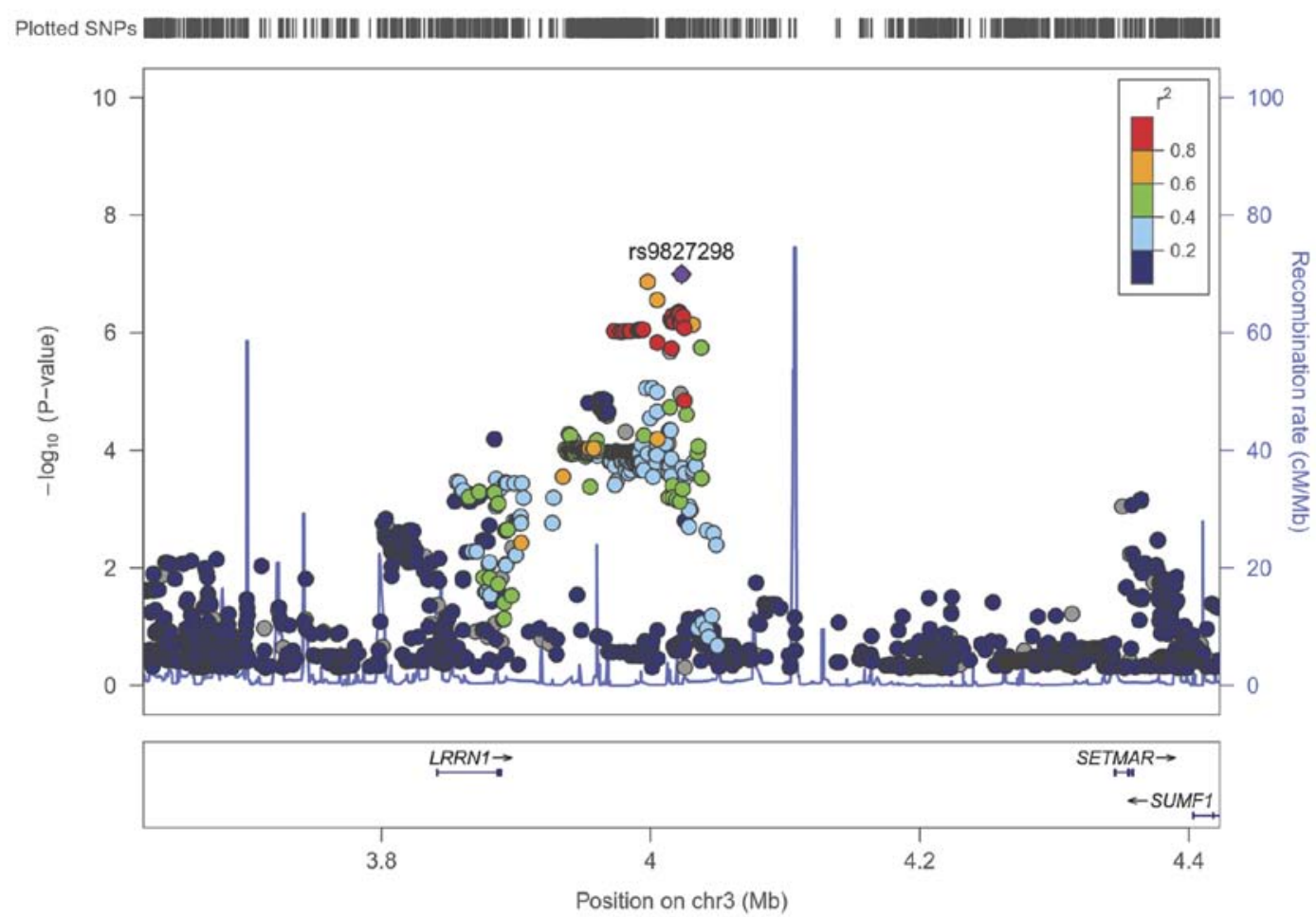

Figure 5. LocusZoom of the rs9827298. Chr3:4023053, $\mathrm{P}=1 \times 10^{-7}$. Each dot represents the P-value of the SNP obtained in the genome-wide association study. The color of the dots represents the linkage disequilibrium $\left(\mathrm{r}^{2}\right)$, which means that the frequency of association of the different alleles is higher than what would be expected if the loci were independent and associated randomly. Chr, chromosome; SNP, single-nucleotide polymorphism; LRRN1, leucine-rich repeat neuronal 1; SETMAR, SET domain and mariner transposase fusion gene; SUMF1, sulfatase-modifying factor 1.

In addition to the associative gene loci, the present study identified six loci that may have an association with fracture risk. The most adjacent genes to the observed locus in chr 3 (lead SNP rs9827298) were LRRN1, SUMF1 and SETMAR. 
Table III. Genetic variants identified in the genome-wide association study for childhood fractures performed in the Northern Finland Birth Cohort 1986.

\begin{tabular}{lrllllllll}
\hline SNP ID & Chr & Position & EA & NEA & EAF & BETA & SE & P-value & Adjacent gene(s) \\
\hline rs112635931 & 10 & 11891165 & A & G & 0.0512 & 3.187 & 0.551 & $7.28 \times 10^{-9}$ & PROSER2, PROSER2-AS1 \\
rs9827298 & 3 & 4023053 & G & C & 0.0635 & 2.325 & 0.437 & $1.01 \times 10^{-7}$ & LRRN1, SETMAR, SUMF1 \\
rs374077976 & 8 & 126506632 & C & CTT & 0.1462 & 1.752 & 0.333 & $1.46 \times 10^{-7}$ & TRIB1, NSMCE2 \\
rs41316954 & 9 & 101767382 & T & C & 0.0890 & 1.809 & 0.375 & $1.38 \times 10^{-6}$ & COL15A1, TGFBR1 \\
rs17762577 & 14 & 97517062 & T & C & 0.4670 & -0.955 & 0.210 & $5.53 \times 10^{-6}$ & VRK1 \\
rs35417231 & 7 & 131772615 & T & C & 0.0703 & 1.988 & 0.412 & $1.37 \times 10^{-6}$ & PLXNA4 \\
rs111299584 & 17 & 69935057 & A & AT & 0.1007 & 1.715 & 0.370 & $3.50 \times 10^{-6}$ & SOX9, SOX9-AS1 \\
\hline
\end{tabular}

Chr, chromosome; EA, effect allele; NEA, non-effect allele; EAF, effect allele frequency; SE, standard error; SNP, single-nucleotide polymorphism; PROSER2-AS1, proline- and serine-rich 2 antisense RNA 1; LRRN1, leucine-rich repeat neuronal 1; SETMAR, SET domain and mariner transposase fusion gene; SUMF1, sulfatase-modifying factor 1; TRIB1, tribbles pseudokinas 1; NSMCE2, NSE2 (MMS21) homolog, SMC5-SMC6 complex SUMO ligase; COL15A1, collagen type XV alpha 1 chain; TGFBR1, transforming growth factor beta receptor 1; VRK1, vaccinia-related kinase 1; PLXNA4, Plexin A4; SOX9, SRY-Box transcription factor 9; SOX9-AS1, SOX9 antisense RNA 1.

Furthermore, based on the GTEx database, it was speculated that rs9827298 may regulate the expression of LRRN1 in the thyroid, lung and blood; however, the database does not include bone tissue or cells. Furthermore, this SNP may affect the SUMF1 gene, which encodes the formylglycine-generating enzyme (FGE). FGE was detected in the endoplasmic reticulum of bone cells, but also in the brain and skin (33). In addition, FGE converts cysteine into $\mathrm{C}$ - $\alpha$-formylglycine and this activates type I sulfatases (52). Previous studies have indicated that there are $\geq 8$ pathologies caused by disruption of sulfatases, including chondrodysplasia punctata type $1(53,54)$. The identified SNP is also in the region of the SETMAR gene, which encodes a fusion protein that binds DNA and functions in DNA repair activities (55). Furthermore, the lead SNP (rs374077976) on chr 8 has previously been associated with total cholesterol (40) and high cholesterol levels have also been suggested to correlate with low BMD in adults $(56,57)$. In addition, there are indications that this may have a relevance in pediatric patients and adolescents (58). The SNP rs41316954 on chr 9 is located within the COL15A1 gene, which encodes the $\alpha$ chain of type XV collagen, a member of the fibril-associated collagens with interrupted helices (FACIT) collagen family. Furthermore, type XV collagen has been revealed to be secreted by human osteoblasts (59). SNP rs111299584 on chr 17 is located upstream of the SOX9 gene, a key transcription factor involved in chondrocyte differentiation. In addition, it has been reported that SOX9 deficiency leads to campomelic dysplasia (60).

A total of two out of the six suggestive loci that were indicated in the GWAS are located in the vicinity of genes that do not have a definitive connection to bone. The first, SNP rs17762577 (chr 14), is located near the vaccinia-related kinase (VRK) serine/threonine kinase 1 gene, which encodes the protein VRK1 (61). The expression of this gene is increased in actively dividing cells and it is widely expressed in cells throughout the body (61). The second, SNP rs35417231 on chr 7, encodes a protein (Plexin-A4, a receptor protein) that is necessary for signaling of semaphorins (62).

The major strengths of the present study are the large size of the birth cohort and the valid fracture data from the
NHDR. Furthermore, the laboratory and statistical methods used to analyze the potential association were validated and of a high standard. Prior to initiation of the present study, it was determined that less severe childhood fractures may not provide reliable information on underlying disorders of bone development. Thus, only fractures treated in hospital were included to provide data with increased reliability regarding the genetic background associated with the risk of fractures. However, the authors acknowledge that in the future, it may be important to perform another study with a population including conventional out-of-hospital treated low-energy fractures and high-energy fractures. Prior to initiation of the study, it was also decided that only fractures among pre-school aged pediatric patients would be included; in later childhood, individual behavioral factors and recreational activities have a greater impact on the individual risk of conventional bone fractures $(63,64)$.

However, there were certain limitations to the present study. For instance, the genetic data were not available for all patients in the cohort, and thus, the study group did not include all the pediatric patients with fractures treated in hospital prior to the age of 7 years. Furthermore, a larger study population with larger amounts of available genetic material would have increased the strength of the GWAS. However, the present sample of 3,230 is still satisfactory for genetic research to assess the preliminary hypothesis of the study. As another limitation, no subgroup analyses were performed, which may have provided further information on differences between sexes or differences between fractures suffered at different ages $(63,64)$. In addition, the number of cases was modest $(n=48)$ and no further sub-group analyses were performed at this stage. Therefore, to validate the present results, they require to be verified in other populations in future studies.

Bone density measurements were not available in the present study. Therefore, it was not possible to perform any analysis regarding BMC or BMD. The fractures that had been caused by a high-energy injury, e.g. a motor vehicle collision, were not excluded, as information on the mechanism of injury was not available for all fractures. The potential effect of the 
injury energy on each individual fracture, as a confounding factor, remains elusive and the results of the present study should be interpreted with this limitation in mind. Furthermore, the healing outcomes of the fractures were not available and this was another limitation of the study.

An additional limitation of the present study is the lack of information regarding the other potential confounding factors in early childhood, including mental or physical health conditions or abnormal weight gain. Furthermore, cases with diseases clearly affecting the bone, including malignancies of the bone and osteogenesis imperfecta, were removed from the study, but other types, e.g. growth retardations, were not excluded. In future studies, it may be useful to consider mental deficits, including Attention Deficit Hyperactivity Disorder or developmental retardations, which may increase the risk of fracture (65). However, two essential factors affecting the pediatric fracture risk in general, sex and age, were assessed in the present analyses.

The nutritional status of pediatric patients over the duration of the in-hospital stay has not been previously documented. In Finland, in general, if a pediatric patient had their first or second fracture and there is an obvious explanation, including a fall, vitamin D levels or other laboratory parameters are not assessed. In cases where chronic disease is suspected, further investigations are performed as a common policy in Finnish hospitals. Among the patients with fracture in the present study, no such cases were present. It should also be noted that vitamin $\mathrm{D}$ supplementation has been provided to Finnish children since the 1940s $(66,67)$. In addition, normal growth and development of all children in Finland are followed up by mandatory and regular nurse or doctor out-of-hospital visits at a maternity clinic from birth until the age of graduation from junior high school (68). Due to these measures, rickets and other illnesses caused by nutritional deficiencies are rare $(66,67)$. In future studies, assessment of the possible implication of the vitamin D levels may be beneficial, as variations in these levels may be a confounding factor.

In future studies it would be beneficial to also take into account recurrent fractures. In the present study only one patient had suffered from >one fracture in the first six years of life and therefore the recurrence of the fractures was not taken into account in the analysis.

To the best of our knowledge, the present study was the first to evaluate the potential association between pediatric fractures and genetics in this particular setting. The present results suggested that genes may have a role in the fracture risk of children, even in cases without a disease affecting the bones. However, further research in this field is required and future studies should be performed with an additional, larger dataset. In addition, further investigations are required to identify the underlying biological mechanisms of the reported association.

\section{Acknowledgements}

The authors would like to acknowledge the biostatistician Mrs. Eeva Vaaramo, M.Sc. (University of Oulu, Faculty of Medicine, Northern Finland Birth Cohort Studies), who was involved in planning the study, and gathering and analyzing the data. The authors would also like to thank Dr Fiona Hanlon-Dearman (University of Alberta, Edmonton AB, Canada) who performed the proofreading of the article.

\section{Funding}

No funding was received.

\section{Availability of data and materials}

The data that support the findings of this study are available from the NFBC (www.oulu.fi/nfbc/), but restrictions apply to the availability of these data, which were used under license for the present study, and so are not publicly available. The remaining datasets used and/or analzyed are available from the corresponding author on reasonable request and with permission of the NFBC.

\section{Authors' contributions}

RP conceived the and designed the present study, and drafted and revised the manuscript. SS acquired, analyses and interpretated the data. SS was also involved in drafting and revising the manuscript. LK was involved in the conception and design of the study, and also took part in the drafting and revising the manuscript. WS was involved in the conception and design of the study as well as revising the manuscript critically. MM was involved in the conception and design of the study. MM also revised the manuscript critically. JJS responsible for the conception and design of the study, and JJS was also involved in drafting and revising the manuscript. All authors read and approved the final manuscript

\section{Ethics approval and consent to participate}

The Ethics Committee of the Northern Ostrobothnia Hospital District approved the study (approval no. 12/2003; 27th of Feb 2003). Participation was voluntary and all participants signed a written informed consent form. Personal information was replaced with an identification code and the data were handled at a group level only. The study followed the principles of the Declaration of Helsinki (Oulu, Finland).

\section{Patient consent for publication}

Not applicable.

\section{Competing interests}

The authors declare that they have no competing interests.

\section{References}

1. Melton LJ III, Atkinson EJ, O'Fallon WM, Wahner HW and Riggs BL: Long-term fracture prediction by bone mineral assessed at different skeletal sites. J Bone Miner Res 8: 1227-1233, 1993.

2. Jones IE, Williams SM, Dow N and Goulding A: How many children remain fracture-free during growth? A longitudinal study of children and adolescents participating in the Dunedin Multidisciplinary Health and Development Study. Osteoporos Int 13: 990-995, 2002. 
3. Manias K, McCabe D and Bishop N: Fractures and recurrent fractures in children; varying effects of environmental factors as well as bone size and mass. Bone 39: 652-657, 2006.

4. Goulding A, Jones IE, Williams SM, Grant AM, Taylor RW, Manning PJ and Langley J: First fracture is associated with increased risk of new fractures during growth. J Pediatr 146: 286-288, 2005

5. Grabala P: Epidemiology of forearm fractures in the population of children and adolescents: Current data from the typical polish city. Orthop Muscular Syst 4: 203, 2015.

6. Ma DQ and Jones G: Clinical risk factors but not bone density are associated with prevalent fractures in prepubertal children J Paediatr Child Health 38: 497-500, 2002.

7. Duren DL, Blangeroc J, Sherwood RJ, Šešelj M, Dyer T, Cole SA, Lee M, Choh AC, Chumlea WC, Siervogel RM, et al Cortical bone health shows significant linkage to chromosomes $2 \mathrm{p}, 3 \mathrm{p}$, and $17 \mathrm{q}$ in 10-year-old children. Bone 49: 1213-1218, 2011.

8. Chesi A, Mitchell JA, Kalkwarf HJ, Bradfield JP, Lappe JM, Cousminer DL, Roy SM, McCormack SE, Gilsanz V, Oberfield SE, et al: A genomewide association study identifies two sex-specific loci, at SPTB and IZUMO3, influencing pediatric bone mineral density at multiple skeletal sites. J Bone Miner Res 32: 1274-1281, 2017.

9. Pocock NA, Eisman JA, Hopper JL, Yeates MG, Sambrook PN and Eberl S: Genetic determinants of bone mass in adults. A twin study. J Clin Invest 80: 706-710, 1987

10. Arden NK, Baker J, Hogg C, Baan K and Spector TD: The heritability of bone mineral density, ultrasound of the calcaneus and hip axis length: a study of postmenopausal twins. J Bone Miner Res 11: 530-534, 1996.

11. Harris M, Nguyen TV, Howard GM, Kelly PJ and Eisman JA Genetic and environmental correlations between bone formation and bone mineral density: a twin study. Bone 22: 141-145, 1998.

12. Mora $S$ and Gilsanz V: Establishment of peak bone mass Endocrinol Metab Clin North Am 32: 39-63, 2003.

13. Goulding A, Jones IE, Taylor RW, Manning PJ and Williams SM: More broken bones: A 4-year double cohort study of young girls with and without distal forearm fractures. J Bone Miner Res 15 2011-2018, 2000.

14. Cook SD, Harding AF, Morgan EL, Doucet HJ, Bennett JT, O'Brien M and Thomas KA: Association of bone mineral density and pediatric fractures. J Pediatr Orthop 7: 424-427, 1987.

15. Kemp JP, Morris JA, Medina-Gomez C, Forgetta V, Warrington NM, Youlten SE, Zheng J, Gregson CL, Grundberg E, Trajanoska K, et al: Identification of 153 new loci associated with heel bone mineral density and functional involvement of GPC6 in osteoporosis. Nat Gen 49: 1468-1475, 2017.

16. Estrada K, Styrkarsdottir U, Evangelou E, Hsu YH, Duncan EL, Ntzani EE, Oei L, Albagha OM, Amin N, Kemp JP, et al: Genome-wide meta-analysis identifies 56 bone mineral density loci and reveals 14 loci associated with risk of fracture. Nat Genet 44: 491-501, 2012.

17. Wynne F, Drummond FJ, Daly M, Brown M, Shanahan F, Molloy MG and Quane KA: Suggestive linkage of 2p22-25 and 11q12-13 with low bone mineral density at the lumbar spine in the Irish population. Calcif Tissue Int 72: 651-658, 2003

18. Karasik D, Cupples LA, Hannan MT and Kiel DP: Age, gender, and body mass effects on quantitative trait loci for bone mineral density: the framingham study. Bone 33: 308-316, 2003.

19. Wilson SG, Reed PW, Bansal A, Chiano M, Lindersson M, Langdown M, Prince RL, Thompson D, Thompson E, Bailey M, et al: Comparison of genome screens for two independent cohorts provides replication of suggestive linkage of bone mineral density to $3 \mathrm{p} 21$ and $1 \mathrm{p} 36$. Am J Hum Genet 72 : 144-155, 2003.

20. Shen H, Zhang YY, Long JR, Xu FH, Liu YZ, Xiao P, Zhao LJ, Xiong DH, Liu YJ, Dvornyk V, et al: A genome-wide linkage scan for bone mineral density in an extended sample: Evidence for linkage on 11q23 and Xq27. J Med Genet 41: 743-751, 2004.

21. Li GH, Cheung CL, Xiao SM, Lau KS, Gao Y, Bow CH, Huang QY, Sham PC and Kung A: Identification of QTL genes for BMD variation using both linkage and gene-based association approaches. Hum Genet 130: 539-546, 2011.

22. Richards JB, Zheng HF and Spector TD: Genetics of osteoporosis from genome-wide association studies: advances and challenges. Nat Rev Genet 13: 576-588, 2012.

23. Goulding A, Cannan R, Williams SM, Gold EJ, Taylor RW and Lewis-Barned NJ: Bone mineral density in girls with forearm fractures. J Bone Miner Res 13: 143-148, 1998
24. Goulding A, Jones IE, Taylor RW, Manning PJ and Williams SM: More broken bones: A 4-year double cohort study of young girls with and without distal forearm fractures. J Bone Miner Res 15 2011-2018, 2000

25. Statistics Finland: Population development in independent Finland-greying Baby Boomers. https://www.stat.fi/tup/suomi90/ joulukuu_en.html. Accessed May 12, 2007.

26. Järvelin MR, Elliott $P$, Kleinschmidt I, Martuzzi M, Grundy C, Hartikainen AL and Rantakallio P: Ecological and individual predictors of birthweight in a northern Finland birth cohort 1986 Paediatr Perinat Epidemiol 11: 298-312, 1997.

27. Northern Finland Cohorts. University of Oulu. https://www.oulu. fi/nfbcl.

28. Schierding W, Antony J, Karhunen V, Vääräsmäki M, Franks S Elliott P, Kajantie E, Sebert S, Blakemore A, Horsfield JA, et al: GWAS on prolonged gestation (post-term birth): Analysis of successive Finnish birth cohorts. J Med Genet 55: 55-63, 2018.

29. Finnish Institute for Health and Welfare: Care Register for Health Care. https://thl.fi/en/web/thlfi-en/statistics/information-on-statistics/ register-descriptions/care-register-for-health-care

30. Parkkari J, Mattila V, Niemi S and Kannus P: Injury-related death among Finnish children, 1971-2001. JAMA 289: 702-703, 2003.

31. World Health Organization: Basic tabulation list with alphabetic index. In: International Classification of Diseases Ninth revision. World Health Organization, Switzerland, 1978.

32. Lander E and Kruglyak L: Genetic dissection of complex traits: guidelines for interpreting and reporting linkage results. Nat Genet 11: 241-247, 1995.

33. Turner SD: qqman: An R package for visualizing GWAS results using Q-Q and manhattan plots. J Open Source Softw 3:731, 2018.

34. Li MJ, Wang P, Liu X, Lim EL, Wang Z, Yeager M, Wong MP, Sham PC, Chanock SJ and Wang J: GWASdb: A database for human genetic variants identified by genome-wide association studies. Nucleic Acids Res 40: D1047-5104, 2012.

35. GWAS Catalog. The NHGRI-EBI Catalog of published genomewide association studies. https://www.ebi.ac.uk/gwas/.

36. GTEx Consortium: Human genomics. The Genotype-Tissue Expression (GTEx) pilot analysis: Multitissue gene regulation in humans. Science 348: 648-660, 2015.

37. Boyle AP, Hong EL, Hariharan M, Cheng Y, Schaub MA, Kasowski M, Karczewski KJ, Park J, Hitz BC, Weng S, et al: Annotation of functional variation in personal genomes using RegulomeDB. Genome Res 22: 1790-1797, 2012.

38. Trajanoska K, Morris JA, Oei L, Zheng HF, Evans DM, Kiel DP, Ohlsson C, Richards JB and Rivadeneira F: Assessment of the genetic and clinical determinants of fracture risk: Genome wide association and mendelian randomisation study. BMJ 362: k3225, 2018.

39. Medina-Gomez C, Kemp JP, Trajanoska K, Luan J, Chesi A, Ahluwalia TS, Mook-Kanamori DO, Ham A, Hartwig FP, Evans DS, et al: Life-course genome-wide association study meta-analysis of total body BMD and assessment of age-specific effects. Am J Hum Genet 102: 88-102, 2018.

40. Asselbergs FW, Guo Y, van Iperen EP, Sivapalaratnam S, Tragante V,Lanktree MB, Lange LA, Almoguera B,Appelman YE, Barnard J, et al: Large-scale gene-centric meta-analysis across 32 studies identifies multiple lipid loci. Am J Hum Genet 91: 823-838, 2012.

41. Parviainen R, Auvinen J, Pokka T, Serlo W and Sinikumpu JJ: Maternal smoking during pregnancy is associated with childhood bone fractures in offspring - A birth-cohort study of 6718 children. Bone 101: 202-205, 2017.

42. Sinikumpu JJ, Pokka T, Sirnio K, Ruuhela R and Serlo W: Population-based research on the relationship between summer weather and paediatric forearm shaft fractures. Injury 44 : 1569-1573, 2013

43. Sinikumpu JJ: Too many unanswered questions in children's forearm shaft fractures: High-standard epidemiological and clinical research in pediatric trauma is warranted. Scand J Surg 104: 137-138, 2015.

44. Pan F, Xiao P, Guo Y, Liu YJ, Deng HY, Recker RR and Deng HW: Chromosomal regions 22q13 and 3p25 may harbor quantitative trait loci influencing both age at menarche and bone mineral density. Hum Genet 123: 419-427, 2008.

45. Ioannidis JP, Ng MY, Sham PC, Zintzaras E, Lewis CM, Deng HW, Econs MJ, Karasik D, Devoto M, Kammerer CM, et al: Meta-analysis of genome-wide scans provides evidence for sexand site-specific regulation of bone mass. J Bone Miner Res 22: 173-183, 2007. 
46. Brunkow ME, Gardner JC, Van NJ, Paeper BW, Kovacevich BR, Proll S, Skonier JE, Zhao L, Sabo PJ, Fu Y, et al: Bone dysplasia sclerosteosis results from loss of the SOST gene product, a novel cystine knot-containing protein. Am J Hum Genet 68: 577-589, 2001.

47. Chesi A, Mitchell JA, Kalkwarf HJ, Bradfield JP, Lappe JM, McCormack SE, Gilsanz V, Oberfield SE, Hakonarson H, Shepherd JA, et al: A trans-ethnic genome-wide association study identifies gender-specific loci influencing pediatric aBMD and BMC at the distal radius. Hum Mol Genet 24: 5053-5059, 2015.

48. Gene Page. https://www.gtexportal.org/home/gene/PROSER2. Accessed March 5, 2019.

49. The Human Protein Atlas. https://www.proteinatlas.org/ ENSG00000148426-PROSER2/tissue. Accessed March 5, 2019.

50. GEO, Gene Expression Omnibus Profile. https://www.ncbi.nlm. nih.gov/geoprofiles/94126705. Accessed March 5, 2019.

51. GEO, Gene Expression Omnibus Profile. https://www.ncbi.nlm nih.gov/geoprofiles/35462505. Accessed March 5, 2019.

52. Dierks T, Schmidt B, Borissenko LV, Peng J, Preusser A, Mariappan M and von Figura K: Multiple sulfatase deficiency is caused by mutations in the gene encoding the human Ca-formylglycine generating enzyme Cell 113: 435-444, 2003.

53. Appel MJ and Bertozzi CR: Formylglycine, a post-translationally generated residue with unique catalytic capabilities and biotechnology applications. ACS Chem Biol 10:72-84, 2015.

54. Diez-Roux G and Ballabio A: Sulfatases and human disease. Annu Rev Genomics Hum Genet 6: 355-379, 2005.

55. GeneCards, Human Gene Database. www.genecards.org/cgi-bin/ carddisp.pl?gene=SETMAR . Accessed March 5, 2019.

56. Mandal CC: High Cholesterol deteriorates bone health: New insights into molecular mechanisms. Front Endocrinol (Lausanne) 6: 165, 2015.

57. Ghosh-Choudhury N, Mandal CC and Choudhury GG: Statin-induced Ras activation integrates the phosphatidylinosito 3-kinase signal to Akt and MAPK for bone morphogenetic protein-2 expression in osteoblast differentiation. J Biol Chem 282: 4983-4993, 2007.

58. Lim HH: Effect of serum cholesterol on bone mineral density in normal-weight children and adolescents. J Pediatr Endocrinol Metab 28: 1313-1319, 2015
59. Lisignoli G, Codeluppi K, Todoerti K, Manferdini C, Piacentini A, Zini N, Grassi F, Cattini L, Piva R, Rizzoli V, et al: Gene array profile identifies collagen type XV as a novel human osteoblast-secreted matrix protein. J Cell Physiol 220: 401-409, 2009.

60. Matsushita M, Kitoh H, Kaneko H, Mishima K, Kadono I, Ishiguro N and Nishimura G: A novel SOX9 H169Q mutation in a family with overlapping phenotype of mild campomelic dysplasia and small patella syndrome. Am J Med Genet 161A: 2528-2534, 2013.

61. GeneCards, Human Gene Database. https://www.genecards.org/ cgi-bin/carddisp.pl?gene=VRK1. Accessed March 5, 2019.

62. Sijaona A, Luukko K, Kvinnsland IH and Kettunen P: Expression patterns of Sema3F, PlexinA4, -A3, Neuropilin1 and -2 in the postnatal mouse molar suggest roles in tooth innervation and organogenesis. Acta Odontol Scand 70: 140-148, 2012.

63. Javaid MK and Cooper C: Prenatal and childhood influences on osteoporosis. Best Pract Res Clin Endocrinol Metab 16: 349-367, 2002.

64. Godfrey K, Walker-Bone K, Robinson S, Taylor P, Shore S, Wheeler T and Cooper C: Neonatal bone mass: Influence of parental birthweight, maternal smoking, body composition, and activity during pregnancy. J Bone Miner Res 16: 1694-1703, 2001.

65. Guo NW, Lin CL, Lin CW, Huang MT, Chang WL, Lu TH and Lin CJ: Fracture risk and correlating factors of a pediatric population with attention deficit hyperactivity disorder: A nationwide matched study. J Pediatr Orthop B 25: 369-74, 2016.

66. Lehtonen-Veromaa M, Möttönen T, Irjala K, Kärkkäinen M, Lamberg-Allardt C, Hakola P and Viikari J: Vitamin D intake is low and hypovitaminosis D common in healthy 9- to 15-year-old Finnish girls. Eur J Clin Nutr 53: 746-751, 1999.

67. Hallman N, Hultin H and Visakorpi JK: Prophylactic use of vitamin d in Finland. Duodecim 80, 185-9, 1964 (In Swedish).

68. Ministry of Social Affairs and Health. Maternity and child health clinics. https://stm.fi/en/maternity-and-child-health-clinics.

This work is licensed under a Creative Commons

Attribution-NonCommercial-NoDerivatives 4.0 International (CC BY-NC-ND 4.0) License. 